Published in final edited form as:

J Chromatogr B Analyt Technol Biomed Life Sci. 2010 January 15; 878(2): 258. doi:10.1016/j.jchromb. 2009.08.038.

\title{
Internally Calibrated Quantification of VEGF in Human Plasma by Fluorescence Immunoassays in Disposable Elastomeric Microfluidic Devices
} \author{
$\underset{1,2}{\text { David H. Lin }}{ }^{1}$, Clive R. Taylor ${ }^{2}$, W. French Anderson ${ }^{3}$, Axel Scherer ${ }^{1}$, and Emil P. Kartalov ${ }^{*}$, \\ Department of Pathology, Keck School of Medicine, University of Southern California, Los Angeles, \\ CA 90033, and Electrical Engineering Department, California Institute of Technology, Pasadena, \\ CA 91125
}

\begin{abstract}
Herein we report on a proof of principle for the reproducible quantification of Vascular Endothelial Growth Factor (VEGF) in human plasma by fluorescence sandwich immunoassays using disposable polydimethylsiloxane (PDMS) microfluidic chips. The system requires 100 times less sample than typical clinical blood tests, while its current quantification limit is established at $4 \mathrm{pM}$. The in-built calibration method of spiking the plasma with known concentrations of commercially available antigen avoids common sources of error and improves the reliability of the test results. The demonstrated technique is important for immunoassay applications in fundamental scientific research and "point-of-care" (POC) biomedical diagnostics. In particular, the system is immediately applicable to microfluidic quantification of VEGF in human plasma in cancer studies.
\end{abstract}

\section{Keywords}

plasma; immunoassay; microfluidic; fluorescence; VEGF; diagnostic

\section{INTRODUCTION}

Today's healthcare is plagued by escalating costs and ever-growing demands on the existing medical infrastructure. The situation will only worsen as the populous Baby Boomer generation approaches retirement age. A potential solution to these challenges is the development of personal, preventative, participatory, and predictive medicine that will combine personalized genomic and proteomic information with systematic monitoring, early diagnosis, timely treatment, and preventative therapies.

A critical component in this emerging healthcare model will be the ubiquitous practice of rapid, accurate, inexpensive, and frequent biomedical testing. However, this task is financially and

\footnotetext{
*to whom the correspondence should be addressed: kartalov@ usc.edu, tel(323) 442-3211, physical address: 2011 Zonal Ave, HMR301A, Keck School of Medicine, University of Southern California, Los Angeles, CA 90089-9092.

${ }_{1}^{1}$ Electrical Engineering Dept, California Institute of Technology, 1200 E California Blvd, Pasadena, CA 91125

${ }^{2}$ Dept of Pathology, Keck School of Medicine, University of Southern California, Los Angeles, California 90033

${ }^{3}$ Dept of Biochemistry, Keck School of Medicine, University of Southern California, Los Angeles, CA 90033

Publisher's Disclaimer: This is a PDF file of an unedited manuscript that has been accepted for publication. As a service to our customers we are providing this early version of the manuscript. The manuscript will undergo copyediting, typesetting, and review of the resulting proof before it is published in its final citable form. Please note that during the production process errors may be discovered which could affect the content, and all legal disclaimers that apply to the journal pertain.
} 
logistically impossible to achieve with the current centralized clinical diagnostics [1,2]. Instead, a viable solution to this problem is offered by new decentralized diagnostics that would expand the techniques of point-of-care testing (POCT) throughout hospitals, doctor's offices, and even into patients' homes (for certain approved screening modalities). This decentralized diagnostics could be based on disposable microfluidic devices operated by portable inexpensive apparatuses.

So far, while a few commercial systems [1] have been developed (e.g. iSTAT and glucometers), the vast potential for decentralization remains untapped as the majority of testing is still conducted in clinical labs using large equipment [2]. Hence the need for such devices remains unsatisfied and so, their development is an active area of research.

In particular, reducing immunoassays to microfluidic scales has been extensively explored in recent years, because many blood tests are protein-based and immunoassays are the chief method of protein detection and quantification. In addition, as new biomarkers are discovered, the need for rapid and accurate quantification of proteins in biosamples will only increase.

Many microfluidic immunoassay approaches have been proposed, involving glass [3-10], titanium dioxide [8], silicon [11-15], silicone [11,16-31], silicon nitride [32], poly (methylmethacrylate) [33], polyurethane [34], Mylar [35], polycarbonate [36], polyolefin [37], ethylenediamine film [38], compact discs [39], flow cells [40], screen-printed chips [41], and scanned arrays [42]. These diverse efforts boast subsets of the full list of the desirable qualities: capability to measure multiple antigens and samples per device, industrially feasible fabrication, parsimony of sample and reagents, adequate sensitivity and specificity, adequate reliability and reproducibility, and robust performance in the field. So far, no particular technique has satisfied all these requirements, so the quest for an optimal approach continues.

As part of this effort, we developed a high-throughput multi-antigen microfluidic system [43] simultaneously quantifying four protein analytes at their clinically relevant levels in buffer solutions. Next, we adapted the system to quantify the same blood proteins in human serum [44]. We showed reliable reproducible quantification of ferritin down to $250 \mathrm{pM}$ endogenous concentration [44], thereby achieving proof of principle for work with human serum.

However, some important analytes, such as Vascular Endothelial Growth Factor (VEGF) (used in the diagnosis and monitoring of cancer $[45,46])$, are measured in plasma instead of serum. Serum production involves letting the whole blood coagulate, centrifuging the sample to remove the clot, and collecting only the supernatant. During this procedure, many analytes are consumed in the clotting process or become trapped in the coagulating matrix. Hence, their concentration in the resulting serum is significantly less than the starting one in whole blood. The reduction factor is generally inconsistent, preventing reliable quantification.

Consequently, such analytes are instead clinically measured in human plasma $[45,46]$.

Hence, we applied our system [44] to the quantification of selected analytes in human plasma. We measured VEGF reproducibly down to $4 \mathrm{pM}$ endogenous concentration. The achieved proof of principle extends the usefulness and applicability of this microfluidic system to work with human plasma at very low analyte concentrations. Our results are presented herein.

The reported work brings the field of microfluidics closer to the desired goal of ubiquitous and affordable decentralized protein-based biomedical diagnostics. The presented approach is particularly relevant and useful because production of plasma can also be done 'onchip' [42] in the same type of elastomeric devices. The technical and architectural compatibility of these two techniques allow the integration of sample preparation and sensitive quantification within the same device, thereby miniaturizing the overall system, speeding up the measurement 
procedure, simplifying logistics, and reducing costs. The eventual result would be a rapid and accurate blood test requiring only a droplet of whole blood.

Finally, the inherent sample economy makes our system ideally suited for applications where plasma samples are small, scarce, or expensive to obtain. Important examples are large studies that monitor and/or screen communities and pediatric populations, as well as broad retrospective studies that span already generated bio-banks of patient samples. Such studies cannot be undertaken by conventional techniques, because those techniques would necessitate sample volumes that are simply unavailable or would be too costly to obtain by orthodox venipuncture. By contrast, the microfluidic chip described herein functions well with just a few microliters of plasma. We expect that such studies will be more easily undertaken in the future, now that an enabling technique has become available.

\section{MATERIALS AND METHODS}

\subsection{Device Fabrication}

Flow layer mold-3-inch silicon wafers were treated with hexadimethylsilazane (HDMS) vapors for $3 \mathrm{~min}$. SPR 220-7 photoresist (MicroChem Corp, Newton, MA) was spun onto the wafer using a WS-400A-GNPP/LITE spincoater (Laurell Technologies, North Wales, PA). The wafer was baked at $105{ }^{\circ} \mathrm{C}$ for $90 \mathrm{sec}$, UV-exposed through a printed transparency mask at a Karl Suss MJB3 mask aligner, and developed in SPR 220-7 developer. The mold was baked on a hotplate at $140{ }^{\circ} \mathrm{C}$ for 30 min with ramping up from and back to room temperature.

Control layer mold-SU8-2010 (MicroChem Corp, Newton, MA) was spun onto 3-inch silicon wafer using the same spin coater. Pre-exposure bake was $2 \mathrm{~min}$ at $65^{\circ} \mathrm{C}$, then $6 \mathrm{~min}$ at $95^{\circ} \mathrm{C}$. UV exposure was done at the same mask aligner for $1.75 \mathrm{~min}$. Post-exposure bake was 2 min at $65^{\circ} \mathrm{C}$, then $6 \mathrm{~min}$ at $95^{\circ} \mathrm{C}$. The mold was developed in SU8 developer (MicroChem Corp, Newton, MA).

Elastomer Chip_Molds were exposed to tetramethylchlorosilane (TCMS) vapors for $3 \mathrm{~min}$. Then 35 and 21 grams of PDMS pre-polymer, in monomer-to-catalyst weight ratio of 5:1 and 20:1 respectively, were stirred and degassing using a HM-501 hybrid mixer (Keyence, Long Beach, CA, USA). The 20:1 mixture was spun onto the flow layer mold at $1500 \mathrm{rpm}$ for 60 sec using a P6700 spincoater (Specialty Coating Systems, Indianapolis, IN, USA). The 5:1 was poured directly onto the control layer wafer. Both were baked in an $80^{\circ} \mathrm{C}$ oven for $30 \mathrm{~min}$. The control layer was peeled off the mold and cut out into devices. Control ports were punched using a 20-gauge Intramedic TM Luer-Stub adapter (BD Biosciences, Franklin Lakes, NJ). The devices were then aligned and assembled to the flow layer under a stereoscope. The result was baked in an $80^{\circ} \mathrm{C}$ oven for $1 \mathrm{hr}$. Devices were cut out and peeled off the flow layer mold. Ports for the flow channels were then punched using the same 20-gauge adapter. The resulting PDMS chips was washed in ethanol, dried with nitrogen, and attached to epoxide-coated glass slides (Arrayit Corporation, Sunnyvale, CA). A final $12 \mathrm{hr}$ bake was performed to bond the PDMS chip to the slide.

\subsection{Sample Preparation}

A compound human plasma sample was produced at the USC Reference Lab by combining the leftover plasma samples from six anonymized patients. A portion of the compound sample was sent to Quest Diagnostics Nichols Institute (San Juan Capistrano, CA) for VEGF quantification by conventional clinical means. The returned result was $42 \mathrm{pg} / \mathrm{mL}$. The rest of the sample was aliquoted and frozen at $-20^{\circ} \mathrm{C}$ for storage. 
For each on-chip experiment, an aliquot would be thawed and split into five samples. Four of them would be spiked with a different concentration of antigen that was a commercial analog to the analyte of interest. The fifth one would remain unspiked. The resulting preparations were fed as separate samples on the same chip, along with a sixth sample (Tris 1X, 0.1\% BSA) for control.

\subsection{Experimental Setup and Procedures}

The microfluidic fluorescence microscopy station contains an inverted Olympus IX-71 fluorescence microscope (Olympus America, Melville, NY) equipped with a mercury lamp (HBO ${ }^{\circledR} 103$ W/2; OSRAM Munich, Germany) and a cooled CCD camera ST-7I (Santa Barbara Instrument Group, Santa Barbara, CA). Microfluidic control is provided by $8 \mathrm{CM}$ solenoid valve arrays and a BOB3 box (Fluidigm Corp., San Francisco, CA) directed by a PCI NI-DAQ card from National Instruments.

23-gauge steel tubes (New England Small Tube, Litchfield, $\mathrm{NH}$ ) are inserted into the control and flow layer ports of the chip (Figure 1A). (As the diameter of the ports is smaller than the outer diameter of the tubes, the chip material is stretched around the tubes and holds them snugly. The resulting seal typically remains airtight up to 25 psi pressure difference.) The control layer is filled with water using Tygon ${ }^{\circledR}$ tubing (Cole-Parmer, Vernon Hills, IL) connected to the $8 \mathrm{CM}$ arrays. The flow layer steel tubes filled with samples and reagents through Tygon $®$ tubing connected to pressure manifolds from Corning. The operating air pressures were maintained at 13 psi for control channels and 8 psi for sample and reagents channels, using regulators from AirTrol.

The chip (Figure 1) is essentially a two-dimensional matrix of analyte capture chambers with added input/output ports for samples, reagents, and pneumatic control. The chambers are formed at the intersections of reagent and sample channels (in blue). The chambers can be isolated from one another by a vertical and a horizontal array of pneumatic microvalves operated through control channels (in red). During each feed, valves are opened or closed to form a fluidic pathway from input to exhaust for only the desired sample or reagent.

In a quantification experiment, VEGF monoclonal antibody (R\&D Systems, Inc., Minneapolis, $\mathrm{MN}$ ) is fed from inputs D1-5 to exhausts DE1-5 (Figure 1B) in 10 cycles of 60-sec flow and 60 -sec incubation. The monoclonal antibody travels along the 5 test lanes (horizontally to the right on Figure 1B) and bonds covalently to the epoxide-coated glass substrate, which forms the floor of the reagent channels. Next, buffer (Tris 1X (Sigma-Aldrich), 0.1\% BSA) is fed from input DB to exhausts DE1-5, to flush the non-bound monoclonal antibodies out of the test lanes and to passivate any unreacted epoxide. Buffer of the same content is then fed from input SB to exhausts SE1-6 vertically downward on the Figure 1B, to passivate the epoxide along the sample paths as well. (The passivation feeds also ensure that BSA blocks nonspecific-binding sites on the elastomer channels walls, which otherwise might later bind fluorescently labeled streptavidin and produce false signal during detection and quantification. Low signal from controls attests to the success of the technique.)

Spiked plasma subsamples are then fed from inputs S1-5 to exhausts SE1-5, while buffer (Tris $1 \mathrm{X}, 0.1 \% \mathrm{BSA}$ ) is simultaneously fed from S6 to SE6 as a control, in 10 cycles of 60-sec flow and 60 -sec incubation. During each incubation, the subsamples are pumped along respective circular paths (coliseums [43,44], Figure 1B) using an array of peristaltic pumps set at a 3 -sec cycle. This technique ensures that the same plasma passes over the capture sites multiple times, thereby maximizing the capture of analyte by the immobilized antibody.

After the plasma feeds, buffer (Trix 1X, 0.1\% BSA) from SB to SE1-6 flushes out the remaining plasma. Biotinylated VEGF polyclonal antibody (R\&D Systems, Inc., Minneapolis, MN) is 
fed from inputs A1-5 to exhausts DE1-5 in 10 cycles of 60 -sec flow and 60-sec incubation. The antibody attaches to the captured analyte and completes the sandwich immunoassay. Buffer (Tris $1 \mathrm{X}, 0.1 \% \mathrm{BSA}$ ) is then fed from input DB to exhausts DE1-5 to flush unbound antibody.

Streptavidin tagged with Alexa Fluor 555 (Invitrogen Corp., Carlsbad, CA) is fed from input SA to exhausts SE1-5 in 10 cycles of 60-sec flow and 60-sec incubation. The streptavidin binds to the immobilized biotinylated antibody. Buffer (Tris 1x, 0.1\% BSA) is fed from DB to DE1-5 for $10 \mathrm{~min}$ to flush the excess streptavidin. The CCD camera is cooled to $-5^{\circ} \mathrm{C}$ and a fluorescence image is taken of each capture chamber in the matrix with a 5 -sec exposure.

\subsection{Data Analysis}

Signal quantification inside fluorescence images was done by drawing boxes in Astra Image and using the inbuilt summation function. The net signal for each image was calculated as the chamber signal diminished by the background signal adjusted by a factor equal to the ratio of the boxed chamber area to the boxed background area. All the net signals from plasma chambers were diminished by the net signal from the respective control chambers along the same test lane in the device. The results were then plotted as a function of spiked concentration. A linear fit $(\mathrm{R}=0.9757, \mathrm{p}=0.0045)$ was produced to obtain the slope of this calibration curve (Figure 2). Dividing the zero-spike signal by the slope produced the endogenous concentration of the analyte. Results from the multiple quantitations of the same compound plasma sample were organized in a scatter plot (Figure 3A) and a histogram (Figure 3B).

\section{RESULTS}

We set out to achieve a proof of principle for the use of our multi-analyte microfluidic fluorescence immunoassays system $[43,44]$ with human plasma. For this purpose, VEGF was chosen as the analyte to quantify, due to its significance as a cancer biomarker, its low typical endogenous concentration ( $2500 \mathrm{pg} / \mathrm{mL}, 96 \mathrm{pM}$ "normally"), and the standing practice of quantifying it in human plasma.

The same in-built recalibration scheme was utilized as in the preceding work [44] involving human serum. In each experiment, an aliquot of a human plasma stock was split into five samples, four of which was spiked with a different concentration of commercial antigen analog, while the fifth was left pure (unspiked). A buffer control was also included as a sixth sample to offer an estimate of the noise. These samples were processed on the chip (see the experimental section for details). A calibration curve (e.g. Figure 2) was plotted for the net fluorescent signal from the plasma samples as a function of the spiking concentration. The net signal from the pure (zero-spike) plasma sample was divided by the slope of the linear fit to the calibration curve, to obtain a measurement of the endogenous analyte concentration.

Each chip (Figure 1A) produced up to five independent measurements of VEGF in the same plasma aliquot, since the microfluidic matrix (Figure 1B) contains five independent test lanes. Results from multiple measurements within the same chip were combined with results from other chips to produce a scatter plot (Figure 3A) and a histogram (Figure 3B). A Gaussian fit to the histogram produced a mean of $3.93 \mathrm{pM}$ and a standard deviation of $1.92 \mathrm{pM}$, while the clinical Quest Diagnostics measurement of the same sample produced a value of $1.6 \mathrm{pM}$ (42 $\mathrm{pg} / \mathrm{mL}$ ). A likely explanation for the discrepancy is presented in the Discussion below.

We observed no visible difference or systematic clustering among subsets of our measurements. The results showed agreement and reproducibility across different test lanes in the same device as well as across different devices, thereby demonstrating the overall 
reproducibility of the system. We believe the chief contributor to the observed quality is the in-built recalibration scheme.

\section{DISCUSSION}

\subsection{Chosen Method}

Herein we aimed to demonstrate a proof of principle for our system working with human plasma. Hence, we could limit ourselves to the described scheme of combining the plasma of several patients into one sample and quantifying that sample in multiple experiments.

Obviously, the scope and requirements for a full clinical validation will include systematic quantification for a very large number of patients in terms of different analytes measured by 'gold standard' techniques, such as automated ELISA [2]. Such an undertaking is beyond the scope of the present study, which is restricted to proof of principle.

\subsection{Peer Comparison}

In the field of immunoassay miniaturization, our system is one of the few to have demonstrated the capability to work with realistic biomedical samples. While it is prudent to debug emerging systems with buffer solutions as a preliminary step, the ultimate challenge is to produce good results with human serum, plasma, cerebrospinal fluid, urine, saliva, etc. With that requirement in place, the selection of demonstrated devices becomes far more limited $\left[11,21,22,25,{ }^{28}, 29\right.$,

$\left.{ }^{32}, 36-38,40,42\right]$. Except for a surface plasmon resonance method [25] (which is expensive and difficult to parallelize and miniaturize), our system is the apparent leader in quantitative sensitivity.

To our knowledge, among the systems working with realistic biological samples, ours and Linder's [28] are the only ones utilizing an internal recalibration as part of every measurement. However, Linder's uses a second fluorophore as an internal standard, which requires "multicolor" fluorescence detection, increasing cost and impeding miniaturization. Our system achieves recalibration and higher sensitivity, while still working with a single type of fluorophore.

\subsection{Significance and Applicability}

The observed difference between the results obtained by standard clinical measurements (1.6 $\mathrm{pM})$ and by our chips $(3.93 \pm 1.92 \mathrm{pM})$ is intriguing. The self-consistence of our results over a significant number of independent measurements $(\mathrm{N}=39)$ suggests that we are observing a real phenomenon. Our system clearly can detect even such low concentration, but the quantitative measurement has a large uncertainty. These features lead us to believe that we are observing the current quantitative sensitivity limit of the system (around $4 \mathrm{pM}$ ).

Based on these results, our system is immediately usable for VEGF quantification in human plasma, since VEGF is usually overexpressed in practice. In addition, there is a large dynamic range between our sensitivity limit (4 pM) and the "normal" value ( $96 \mathrm{pM})$, in which the system can quantify underexpression, e.g. brought about by VEGF-reducing anticancer drugs [ 45 , 46].

The significance of the presented proof of principle goes beyond VEGF quantification. The proof makes the system applicable to a broad range of clinical diagnostic tests that boil down to quantifying proteins in human plasma. Thus all such tests currently done in macro-samples could instead be done by the presented microfluidic technique, saving reagents and using micro-samples. 
The reduction in required sample volume would allow new types of clinical and fundamental studies, e.g. a broad, multi-analyte screening of a large number of small-volume samples from existing bio-banks organized by the respective symptomatic pathologies, e.g. multiple sclerosis, particular types of cancer, etc. For example, patients' histories could be correlated with the results from the microfluidic testing of their banked samples, to discover new pathological expression signatures of high diagnostic and/or predictive value.

Such studies are usually impractical to conduct by conventional means, due to prohibitive cost and sample volume requirement [2]. However, our system has the inherent capability of multianalyte detection [43,44], which would cut costs, while the system would also use up only a small fraction of the precious banked sample. Hence our system solves both problems. We therefore hope and expect that such valuable studies would be undertaken shortly, now that an enabling technique has been demonstrated.

Apart from quantifications in fundamental studies, our system has relevance to routine biomedical diagnostics as well. On-chip sample preparation is a necessary stage in the highly integrated immunoassay chip of the POC diagnostics of the future. On the other hand, plasma is easier to produce on-chip than serum. Hence, the preferred medium in future POCT would likely be plasma. That makes it expedient to develop subsystems for microfluidic protein quantification in plasma. Our system is an example of this type of development. As an added benefit, our system is already fully integrable with microfluidic devices for plasma preparation $[42,47]$, due to the shared underlying elastomeric microfluidic technology [48]. Thus our system is an important addition to the technological palette necessary to assemble the future of POC diagnostics.

\section{CONCLUSIONS}

The presented work demonstrates proof of principle for the use of our microfluidic fluorescence immunoassay system with human plasma. Vascular Endothelial Growth Factor (VEGF) has been quantified down to $4 \mathrm{pM}$ endogenous concentration, which appears to be the current quantitative sensitivity limit of the system. The demonstrated technique is important for immunoassay applications in scientific research and "point-of-care" biomedical diagnostics. In particular, the system is immediately applicable to microfluidic quantification of VEGF in human plasma in cancer studies.

\section{Acknowledgments}

The authors thank the USC Reference Laboratory for providing the plasma samples. The authors also thank Caltech Kavli Nanoscience Institute and the Caltech Microfluidics Foundry for providing access to their nanofabrication facilities. Financial support was provided by NIH grant R01-HG0026440, NIH grant 1K99007151, NIH grant 4R00EB007151-03, and the Caltech Summer Undergraduate Research Fellowship (SURF) program.

\section{References}

1. Gilbert HC, Szokol JW. Int Anesthesiol Clin 2004;42(2):73. [PubMed: 15087742]

2. Kartalov EP. J In-Vitro Diagnostics Technol. September;2006

3. Wang J, Ibanez A, Chatrathi MP, Escarpa A. Anal Chem 2001;73:5323. [PubMed: 11721936]

4. Fruetel JA, Renzi RF, VanderNoot VA, Stamps J, et al. Electrophoresis 2005;26:1144. [PubMed: 15704246]

5. Angenendt P, Glockler J, Konthur Z, Lehrach H, Cahill DJ. Anal Chem 2003;75:4368. [PubMed: 14632038]

6. Delehanty JB, Ligler FS. Anal Chem 2002;74:5681. [PubMed: 12433105]

7. Sapsford KE, Charles PT, Patterson CH Jr, Ligler FS. Anal Chem 2002;74:1061. [PubMed: 11924964]

8. Sydor JR, Scalf M, Sideris S, Mao GD, et al. Anal Chem 2003;75:6163. [PubMed: 14615996] 
9. Holmes D, She JK, Roach PL, Morgan H. Lab Chip 2007;7:1048. [PubMed: 17653348]

10. Herr AE, Throckmorton DJ, Davenport AA, Singh AK. Anal Chem 2005;77:585. [PubMed: 15649057]

11. Wolf M, Juncker D, Michel B, Hunziker P, Delamarche E. Biosens Bioelectron 2004;19:1193. [PubMed: 15046750]

12. Yakovleva J, Davidsson R, Lobanova A, Bengtsson M, et al. Anal Chem 2004;74:2994. [PubMed: 12141657]

13. Chandrasekaran A, Acharya A, You JL, Soo KY, et al. Sensors 2007;7:1901.

14. Wang ZH, Meng YH, Ying PQ, Qi C, Jin G. Electrophoresis 2006;27:4078. [PubMed: 17054092]

15. Misiakos K, Kakabakos SE, Petrou PS, Ruf HH. Anal Chem 2004;76:1366. [PubMed: 14987094]

16. Delamarche E, Bernard A, Schmid H, Michel B, Biebuyck H. Science 1997;276:779. [PubMed: 9115199]

17. Eteshola E, Balberg M. Biomed Microdevices 2004;6(1):7. [PubMed: 15307439]

18. Phillips KS, Cheng Q. Anal Chem 2005;77:327. [PubMed: 15623312]

19. Piyasena ME, Buranda T, Wu Y, Huang J, et al. Anal Chem 2004;76:6266. [PubMed: 15516117]

20. Kanda V, Kariuki JK, Harrison DJ, McDermott MT. Anal Chem 2004;76:7257. [PubMed: 15595867]

21. Sia SK, Linder V, Parviz BA, Siegel A, Whitesides GM. Angew Chem Int Ed 2004;43:498.

22. Jiang X, Ng JMK, Stroock AD, Dertinger SKW, Whitesides GM. J Am Chem Soc 2003;125:5294. [PubMed: 12720439]

23. Herrmann M, Veres T, Tabrizian M. Lab Chip 2006;6:555. [PubMed: 16572219]

24. Herrmann M, Roy E, Veres T, Tabrizian M. Lab Chip 2007;7:1546. [PubMed: 17960284]

25. Kurita R, Yokota Y, Sato Y, Mizutani F, Niwa O. Anal Chem 2006;78:5525. [PubMed: 16878891]

26. Liu YJ, Guo SS, Zhang ZL, Huang WH, et al. J Appl Phys 2007;102:084911.

27. Sui G, Wang J, Lee CC, Lu W, et al. Anal Chem 2006;78:5543. [PubMed: 16878894]

28. Linder V, Verpoorte E, de Rooij NF, Sigrist H, Thormann W. Electrophoresis 2002;23:740. [PubMed: 11891707]

29. Lin FYH, Sabri M, Erickson D, Alirezaie J, et al. Analyst 2004;129:823. [PubMed: 15343397]

30. Nashida N, Satoh W, Fukuda J, Suzuki H. Biosens Bioelectron 2007;22:3167. [PubMed: 17383171]

31. Lucas LJ, Chesler JN, Yoon JY. Biosens Bioelectron 2007;23:675. [PubMed: 17869502]

32. Murphy BM, He X, Dandy D, Henry CS. Anal Chem 2008;80:444. [PubMed: 18092765]

33. Kim SH, Yang Y, Kim M, Nam SW, et al. Adv Funct Mater 2007;17:3493.

34. Bai Y, Koh CG, Boreman M, Juang YJ, et al. Langmuir 2006;22:9458. [PubMed: 17042569]

35. Nelson KE, Foley JO, Yager P. Anal Chem 2007;79:3542. [PubMed: 17437332]

36. Pugia MJ, Blankenstein G, Peters RP, Profitt JA, et al. Clin Chem 2005;51(10):1923. [PubMed: 16055433]

37. Bhattacharyya A, Klapperich CM. Biomed Microdevices 2006;9(2):245. [PubMed: 17165125]

38. Liang K, Mu W, Huang M, Yu Z, Lai Q. Biomed Microdevices 2007;9(3):325. [PubMed: 17195106]

39. Honda N, Linberg U, Andersson P, Hoffmann S, Takei H. Clin Chem 2005;51(10):1955. [PubMed: 16081503]

40. Mulvaney SP, Cole CL, Kniller MD, Malito M, et al. Biosens Bioelectron 2007;23:191. [PubMed: 17532619]

41. Dong H, Li CM, Zhang YF, Cao XD, Gan Y. Lab Chip 2007;7:1752. [PubMed: 18030397]

42. Fan R, Vermesh O, Srivastava A, Yen BKH, Qin L, Ahmad H, Kwong GA, Liu CC, Gould J, Hood L JR. Heath Nature Biotechnol 2008;26(12):1373.

43. Kartalov EP, Zhong JF, Scherer A, Quake SR, et al. BioTechniques 2006;40(1):85. [PubMed: 16454045]

44. Kartalov EP, Lin DH, Lee DT, Anderson WF, Taylor CR, Scherer A. Electrophoresis 2008;29:5010. [PubMed: 19130581]

45. Hotz HG, Hines OJ, Masood R, Hotz B, Foitzik T, Buhr HJ, Gill PS, Reber HR. Surgery 2005;137 (2):192. [PubMed: 15674201] 
46. Levine AM, Tulpule A, Quinn DI, Gorospe G, Smith DL, Hornor L, Boswell WD, Espina BM, Groshen SG, Masood R, Gill PS. J Clin Oncol 2006;24:1712. [PubMed: 16520466]

47. VanDelinder V, Groisman A. Anal Chem 2006;78:3765. [PubMed: 16737235]

48. Kartalov EP, Anderson WF, Scherer A. J Nanosci Nanotechnol 2006;6(8):2265. [PubMed: 17037833] 


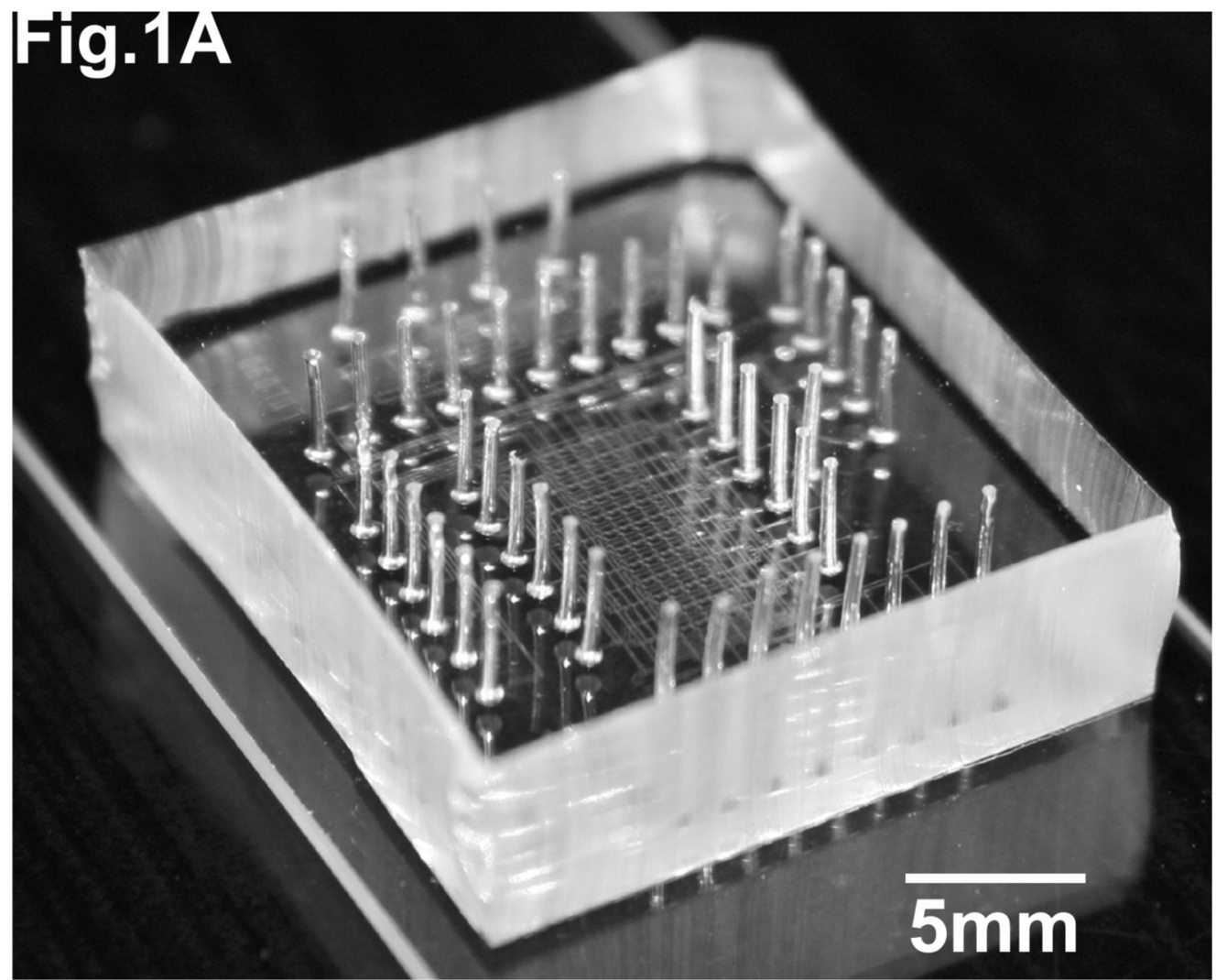

Fig. 1B

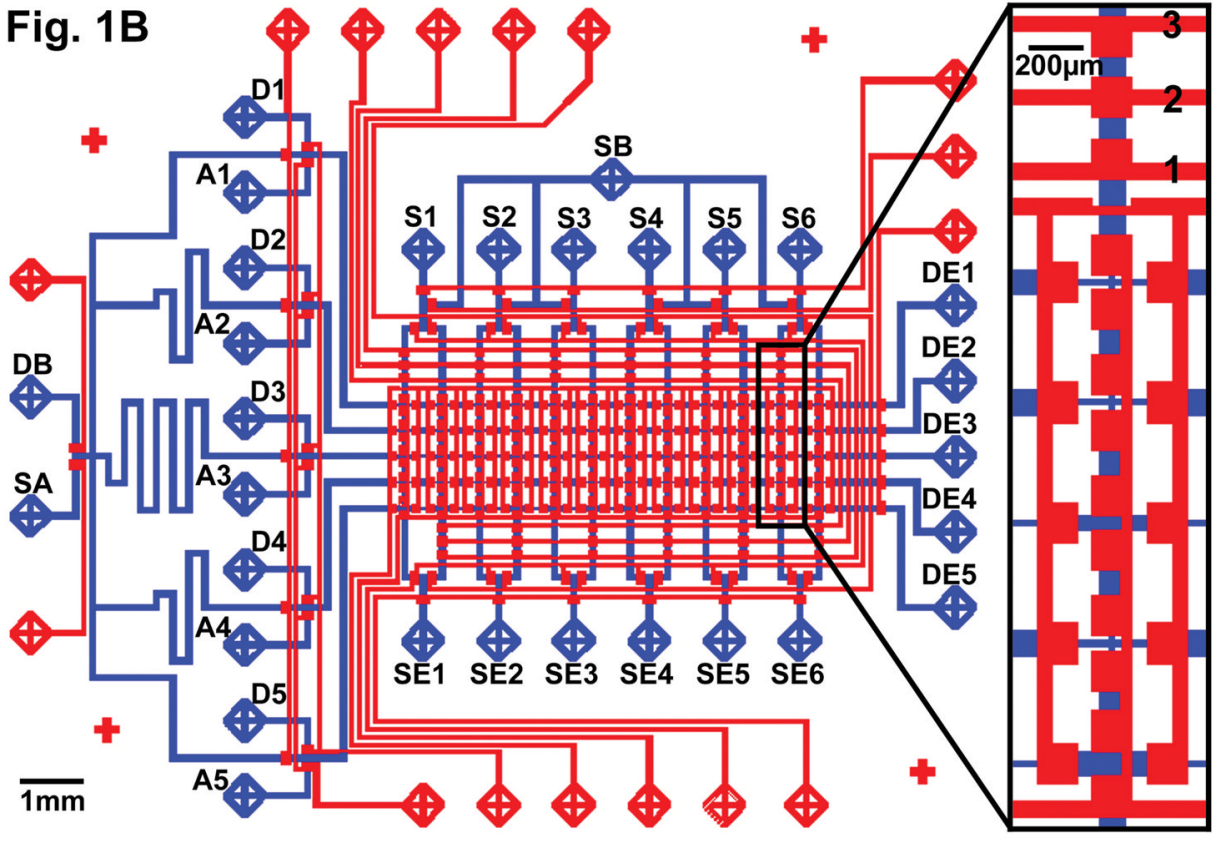

Figure 1.

(A) Microfluidic immunoassay chip. A 60-chamber PDMS chip bound to a one-inch-wide epoxide slide was used for the experiments. The vertical cylinders are input ports for reagents, samples, and control pressure. The microchannel test matrix is visible in the middle. (B)

Architectural diagram of the chip. Control channels (red) convey pressure to open and close 
microvalves, which steer reagents along flow channels (blue). Each intersection of flow channels in the central test matrix forms a microchamber where a sandwich immunoassay is constructed. Fluorophore labels produce signal that is used to quantify the captured antigen. 


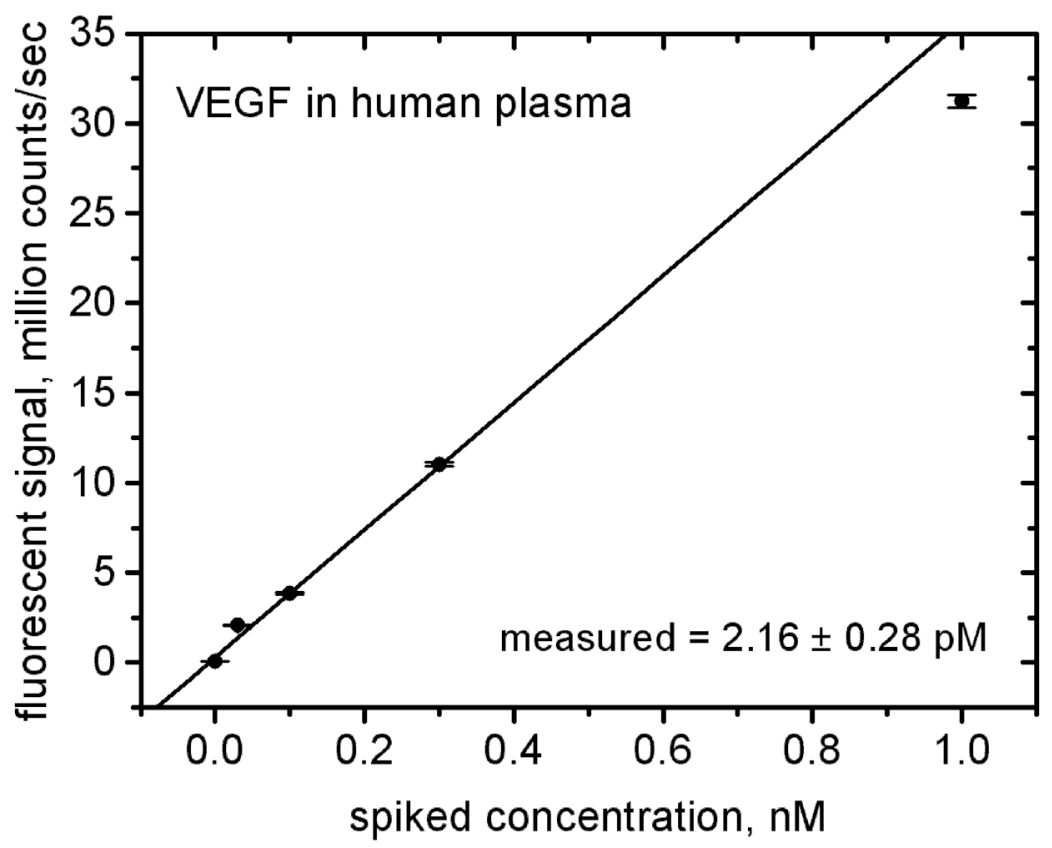

Figure 2. Quantification of VEGF in human plasma

The plasma sample was spiked with known concentrations of commercially available analog. The endogenous VEGF concentration is calculated from the slope of the linear fit and the zerospike net signal. Here, the linear fit $(\mathrm{R}=0.9757, \mathrm{p}=0.0045)$ indicated a concentration of $2.16 \pm$ $0.28 \mathrm{pM}$, while the clinical result was $1.6 \mathrm{pM}$. 


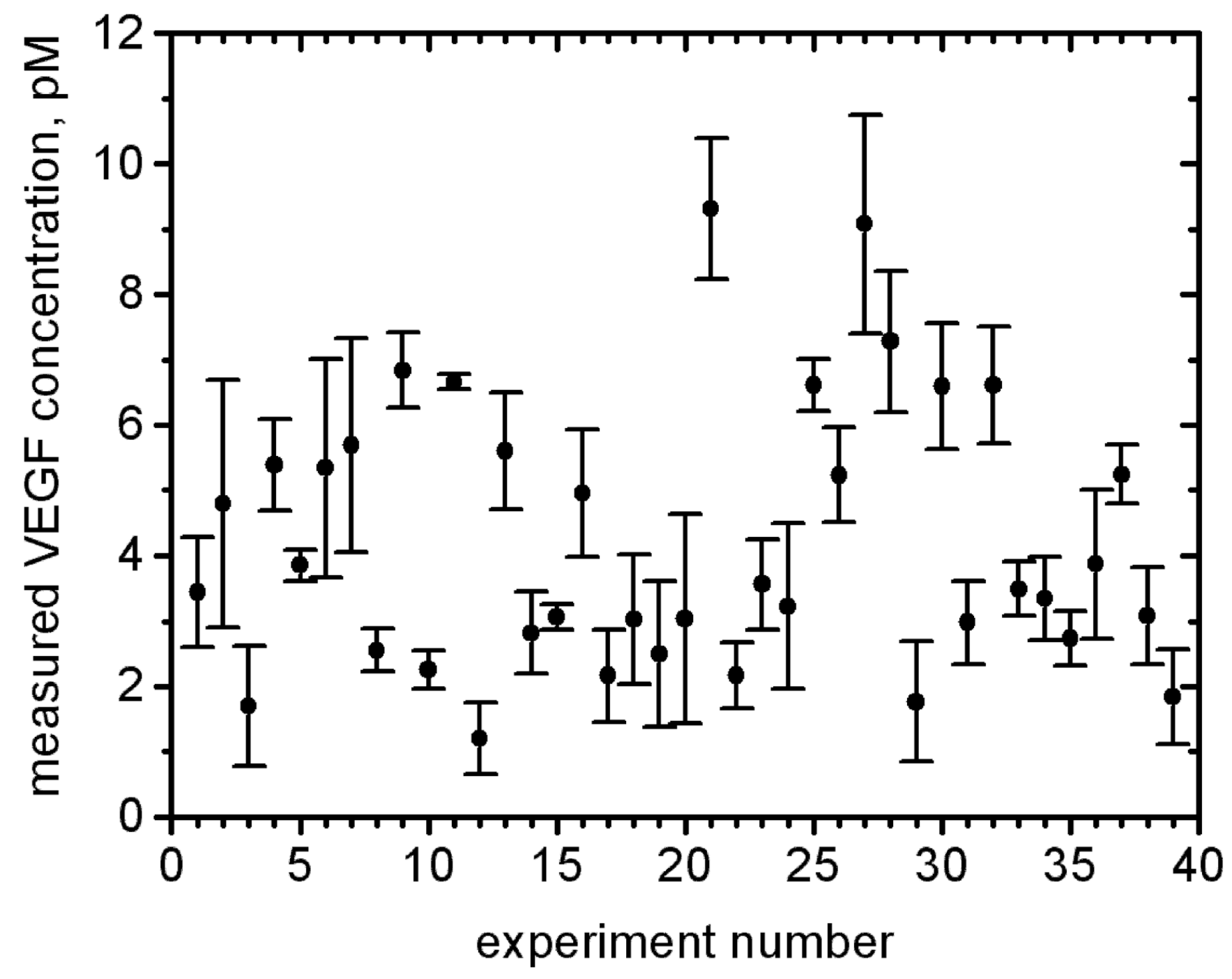




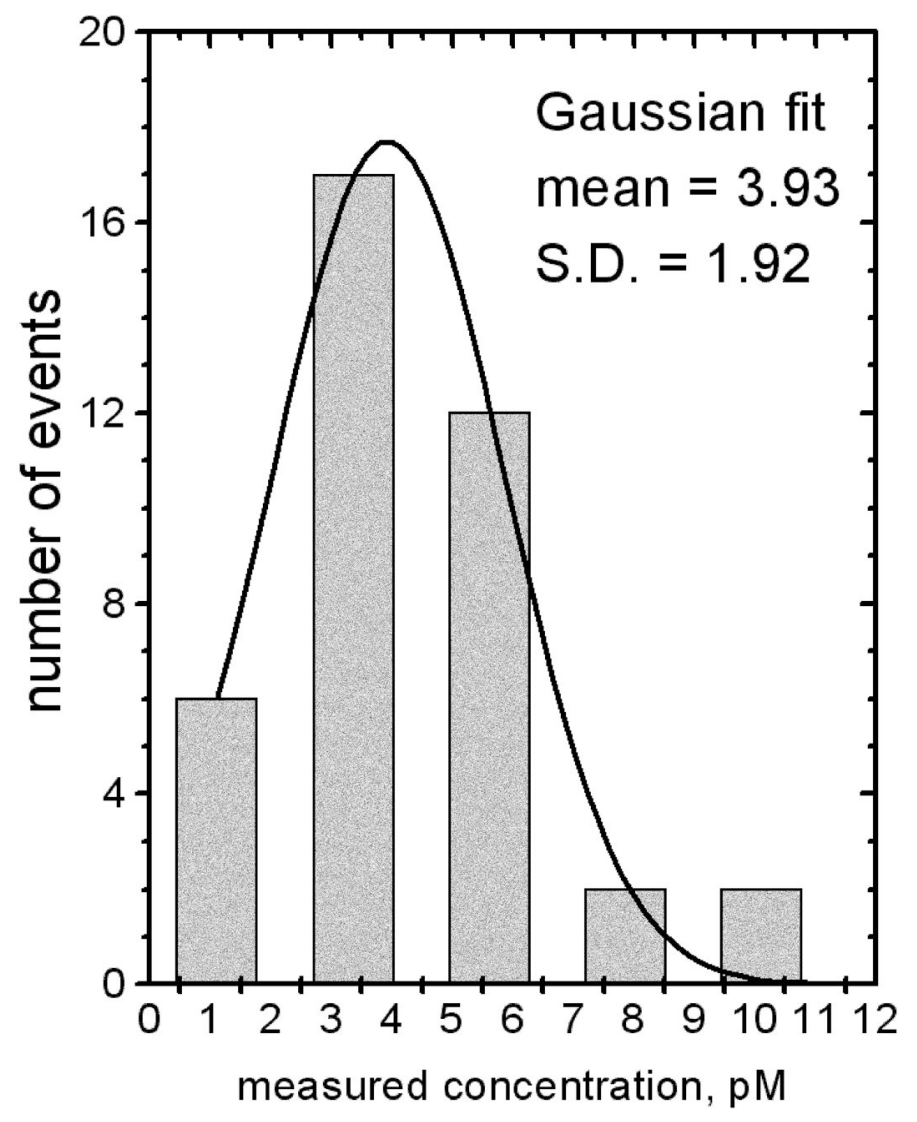

Figure 3. Instrumental Performance

The results of 39 analogous measurements of VEGF in the same human plasma sample are presented as a scatter plot (A) and a histogram (B). No systematic clustering among subsets of measurements was observed. The results showed agreement and reproducibility across different test lanes in the same chip as well as across different chips, thereby attesting to the overall reproducibility of the results. 